\title{
Global parameters of Type Ia supernovae
}

\author{
Bruno Leibundgut ${ }^{1}$ and Maximilian Stritzinger ${ }^{2}$ \\ ${ }^{1}$ ESO, Karl-Schwarzschild-Strasse 3, D-85748 Garching, Germany \\ email: bleibundgut@eso.org \\ ${ }^{2}$ Dark Cosmology Centre, Niels Bohr Institute, University of Copenhagen, \\ Juliane Maries Vej 30, DK-2100 Copenhagen Ø, Denmark \\ email: max@dark-cosmology.dk
}

\begin{abstract}
Using simple physical assumptions we derived fundamental parameters including: the mass of synthesised ${ }^{56} \mathrm{Ni}$, the ejecta mass, and the explosion energy of Type Ia supernovae. The methods have been described in several recent publications and comparison with other methods shows general consistency.
\end{abstract}

Keywords. supernovae: general, stars: evolution, stars: fundamental parameters

Our understanding of thermonuclear supernovae is mostly shaped by the forward comparison of model calculations with observations. While the observational record is normally described in a comparative manner between individual events, the physical parameters governing the explosions have not been directly derived. Explosion models start from very basic and essentially untested assumptions about the progenitor mass, the explosion energy, and the amount of ${ }^{56} \mathrm{Ni}\left(M_{\mathrm{Ni}}\right)$ produced in the explosion. Considering the importance of Type Ia supernovae (SNeIa) for cosmology and for the metal enrichment of the universe, it is important to justify these parameters. As exemplified in the contribution by Howell et al. (2006), surprises are not excluded.

We investigated the explosion characteristics of SNe Ia through the detailed study of their bolometric light curves. The governing factors for their light curve shapes are $M_{\mathrm{Ni}}$, the $\gamma$-ray opacity, the total ejecta mass, and the explosion energy. For a small sample of well-observed SNe Ia we have determined $\mathrm{M}_{\mathrm{Ni}}$ (Stritzinger \& Leibundgut 2005). Our method has been tested by closing the circle from explosion simulations through the radiative transport calculations to synthetic light curves from which $M_{\mathrm{Ni}}$ was derived to within $15 \%$ of the $M_{\mathrm{Ni}}$ in the input model (Blinnikov et al. 2006). Assuming all calculations in this chain were correct, a small correction to the determination of the peak bolometric flux has to be applied to use Arnett's rule (Arnett 1982) to find $M_{\mathrm{Ni}}$ for individual events. Comparison of $M_{\mathrm{Ni}}$ derived from late-phase observations of the iron emission lines (and a density structure taken from models) with results obtained via Arnett's rule yields a consistent picture (Stritzinger et al. 2006b). Current models typically predict smaller amounts of $M_{\mathrm{Ni}}$ than what is derived from observations for most SNe Ia. There is also a wide range of $M_{\mathrm{Ni}}$ produced in these explosions. Simple arguments also yield a lower limit for the Hubble constant - found to be $50 \mathrm{~km} \mathrm{~s}^{-1} \mathrm{Mpc}^{-1}$ (Stritzinger \& Leibundgut 2005).

The late-time evolution of the bolometric light curves were used to estimate the total ejecta mass for a number of SNe Ia. The ejecta mass is a function of both the opacity and explosion energy, as expressed through their expansion velocity (Stritzinger et al. 2006a; see Fig. 1). Again, there is a rather large range of ejecta masses. None of our objects show the canonical Chandrasekhar mass typically assumed for these thermonuclear explosions (Stritzinger et al. 2006a). 


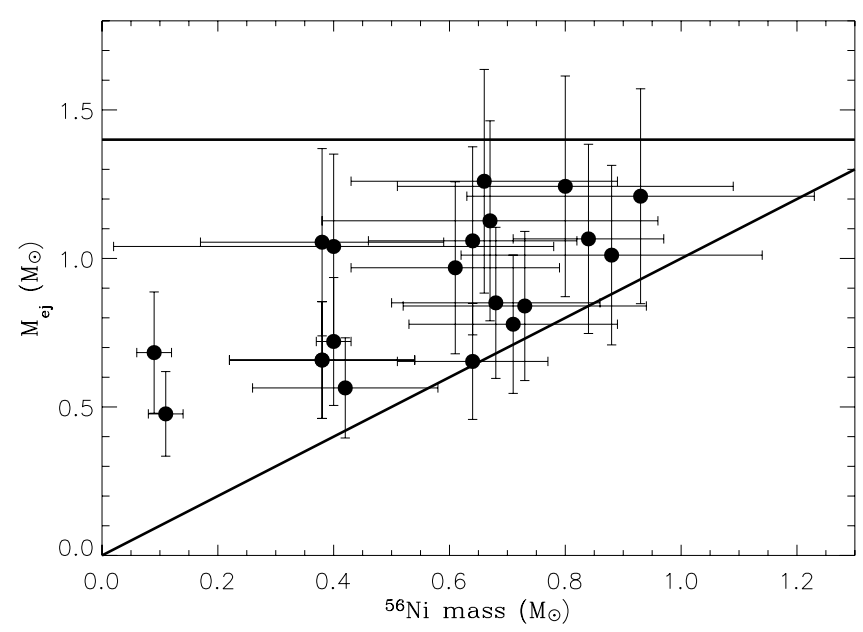

Figure 1. The derived nickel and ejecta masses for a sample of well-observed nearby supernovae. There is a significant scatter amongst the measured masses. While the spread in nickel masses is most likely due to different conditions in the explosions, the scatter in ejecta masses is not understood. Taken from Stritzinger et al. (2006a).

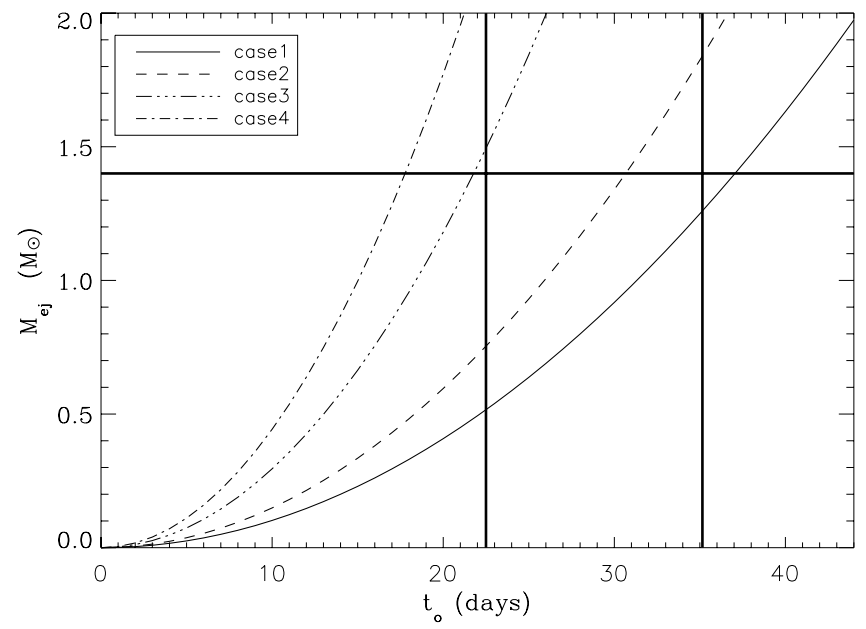

Figure 2. The dependencies of the derived ejecta masses on the assumed $\gamma$-ray opacity and the explosion energy inferred from the expansion velocity. Details can be found in Stritzinger et al. (2006a).

\section{References}

Arnett, W. D. 1982, ApJ, 253, 785

Blinnikov, S., Röpke, F. K., Sorokina, E., I., Gieseler, M., Reinecke, M., Travaglio, C., Hillebrandt, W., \& Stritzinger, M. 2006, A\&A A, 453, 229

Howell, A., et al. 2006, these proceedings

Stritzinger, M., \& Leibundgut, B. 2005, A\&SA, 431, 423

Stritzinger, M., Leibundgut, B., Walch, S., \& Contardo, G. 2006a, A\&A, 450, 241

Stritzinger, M., Mazzali, P., Sollerman, J., \& Benetti, S. 2006b, A\&\&A, 460, 793 